OPEN ACCESS

Edited by:

Guiping Guan,

Hunan Agricultural University, China

Reviewed by:

Qingbiao Xu,

Huazhong Agricultural University,

China

Xin Zong,

Zhejiang University, China

*Correspondence:

Zhenlong Wu

bio2046@hotmail.com

tThese authors have contributed equally to this work and share first authorship

Specialty section:

This article was submitted to

Redox Physiology,

a section of the journal

Frontiers in Physiology

Received: 18 June 2021

Accepted: 26 July 2021

Published: 09 September 2021

Citation:

Chen J, Zhou X, Jia H and Wu Z (2021) Serine Supplementation

Alleviates Doxorubicin-Induced

Oxidative Damage in Skeletal

Muscle of Mice.

Front. Physiol. 12:727093.

doi: 10.3389/fphys.2021.727093

\section{Serine Supplementation Alleviates Doxorubicin-Induced Oxidative Damage in Skeletal Muscle of Mice}

\author{
Jingqing Chen ${ }^{1,2+}$, Xihong Zhou ${ }^{1,3 t}$, Hai Jia ${ }^{1}$ and Zhenlong $W_{u^{1 *}}$ \\ 'State Key Laboratory of Animal Nutrition, China Agricultural University, Beijing, China, 'Laboratory Animal Center of the \\ Academy of Military Medical Sciences, Beijing, China, ${ }^{3}$ Key Laboratory of Agro-ecological Processes in Subtropical Region, \\ Institute of Subtropical Agriculture, Chinese Academy of Sciences, Changsha, China
}

Muscle weakness affects physical activity and quality of life of patients. Serine, a nutritionally non-essential amino acid has been reported to enhance protein synthesis and implicate in biosynthesis of multiple bioactive molecules. It remains unknown whether it can protect mice against oxidative stress-induced muscles weakness. This study was conducted to test the hypothesis that serine administration alleviates doxorubicin-induced oxidative damage in skeletal muscle of mice. Mice pre-treated with or without serine were intraperitoneally injected with either doxorubicin or equal volume of saline. Reactive oxygen species (ROS) accumulation, activity of antioxidant enzymes, oxidation product of protein, DNA, and lipid, activity of mitochondrial complex, and protein level of nuclear-factorerythroid-2-related factor 2 (NRF2)/constitutive-androstane-receptor (CAR) signaling in skeletal muscle of mice were determined. Compared with the control, doxorubicin exposure led to oxidative damage as shown by increased ROS accumulation, decreased activity of antioxidant enzymes, and enhanced oxidative product of protein, DNA, and lipid in the skeletal muscle of mice. These effects of doxorubicin were associated with increased activity of complex I and reduced glutathione. Interestingly, doxorubicin-induced oxidative damage was alleviated by serine administration. Further study showed that the beneficial effect of serine was associated with enhanced NRF2/CAR signaling. Our result showed that serine attenuated doxorubicin-induced muscle weakness in mice. Serine supplementation might be a nutritional strategy to improve the function of skeletal muscle in patients exposed to doxorubicin.

Keywords: doxorubicin, mitochondria, oxidative stress, serine, skeletal muscle

\section{INTRODUCTION}

Doxorubicin is a widely-used chemotherapeutic agent for a variety of tumors in patients (Smuder et al., 2011). Application of doxorubicin has been reported to be associated with muscles weakness and fatigue in patients (Yamamoto et al., 2008; van Norren et al., 2009). These side effects affect quality of life and physical activity, and result in restricted treatment efficacy and poor prognosis. The myotoxicity of doxorubicin is mainly mediated by oxidative damage of proteins, lipid, and DNA (Schwartz, 2000), due to increased production of reactive oxygen species (ROS) in mitochondria of skeleton muscle following doxorubicin administration 
(Smuder et al., 2011; Gilliam et al., 2013). However, therapeutic strategies to alleviate doxorubicin-induced skeleton muscle dysfunction are not available yet.

The glutathione (GSH) is one of the well-known endogenous antioxidants with ability to scavenge ROS, including reactive species, therefore maintaining intracellular homeostasis (Gaucher et al., 2018). Depletion of intracellular GSH has been reported to be associated with ROS accumulation and oxidative damage (Gaucher et al., 2018), which can be reversed by overexpression of $\gamma$-glutamylcysteine ligase $(\mathrm{Gcl})$, a ratelimiting enzyme for GSH synthesis (Meister and Anderson, 1983). Importantly, clinical studies show that patients receiving doxorubicin treatment have a decreased intracellular GSH level and skeleton muscle dysfunction, as compared with the non-treatment individuals (Min et al., 2015; Songbo et al., 2019), indicating that boosting GSH content might be a potentially therapeutic target in ameliorating doxorubicininduced muscle dysfunction.

L-Serine (serine) has traditionally been considered as a nutritionally nonessential amino acid due to its de novo synthesis. Serine plays an essential role in various cellular processes, such as one carbon unit metabolism, synthesis of glycine and cysteine, as well as cellular survival (Kalhan and Hanson, 2012; Metcalf et al., 2018). Offspring from dams fed with a serine deficient diet is vulnerable to oxidative stress (He et al., 2018), indicating a requirement for serine to exert an anti-oxidative capacity. Further studies show that the beneficial effect of serine on muscle weakness is associated with restoration of redox status in animals (Sim et al., 2015; Zhou et al., 2017a,b, 2018a; Cao et al., 2018; He et al., 2018) or various types of cells (Zhou et al., 2018b,c). In the present study, we hypothesized that serine pre-administration prevented doxorubicin-induced oxidative damage of muscle in mice. This hypothesis was based on the observation that doxorubicin exposure lead to a reduce cysteine, a substrate for synthesis of GSH in skeletal muscles (Fabris and MacLean, 2018), which might contribute to reduced GSH level and oxidative damage in the skeleton muscle and other tissues of humans and rodents. Furthermore, serine serves as a metabolic substrate for synthesis of glycine and cysteine, which can be used for GSH synthesis, catalyzed by $\mathrm{Gcl}$, a rate-limiting enzyme (Kalhan and Hanson, 2012; Gaucher et al., 2018), therefore restoring intracellular redox homeostasis.

\section{MATERIALS AND METHODS}

\section{Animal and Diet}

The experimental protocol was approved by the Protocol Management and Review Committee of the Institute of

\footnotetext{
Abbreviations: 8-OHdG, 8-hydroxy-2'-deoxyguanosine; CAR, Constitutiveandrostane-receptor; CAT, Catalase; CuZnSOD, Copper-zinc superoxide dismutase; Gcl, $\gamma$-glutamylcysteine ligase; GPX, Glutathione peroxidase; GRD, Glutathione reductase; GSH, Reduced glutathione; GSSG, Oxidized glutathione; GST, Glutathione S-transferase; Keap1, Kelch like ECH-associated protein 1; MDA, Malondialdehyde; MnSOD, Manganese superoxide dismutase; NRF2, Nuclear-factor-erythroid-2related factor 2; ROS, Reactive oxygen species.
}

Subtropical Agriculture, the Chinese Academy of Science (Changsha, China). A total of 32 6-week-old male C57BL/6 mice were purchased from SLAC Laboratory Animal Central (Changsha, China), and raised in an environment with a temperature set at $22^{\circ} \mathrm{C}$ and a $12 / 12 \mathrm{~h}$ light-dark cycle. Purified AIN93 diet as previous described (Reeves et al., 1993) and water were provided ad libitum. After a 7-day adaptive period, mice were randomly divided into four groups: CON (control), serine (orally administered with serine, $1.5 \mathrm{~g} \mathrm{~kg} \mathrm{BW}^{-1} \mathrm{day}^{-1}$ for 4 weeks), doxorubicin (20 $\mathrm{mg} \mathrm{kg} \mathrm{BW}^{-1}$, i.p.), or Ser-Dox (serine supplementation, and then were subjected to doxorubicin treatment). The dose of doxorubicin used in the present study was based on previous study showing that doxorubicin administration results in oxidative damage in skeletal muscle of mice (Smuder et al., 2011; Kavazis et al., 2014; Min et al., 2015). Forty-eight hours after doxorubicin treatment, all the animals were euthanized by cervical dislocation; skeletal muscles from the upper hindlimb were collected for later analysis.

\section{Preparation of Whole-Muscle Lysates}

For the preparation of whole-muscle lysates, skeletal muscles from the upper hindlimb were homogenized in ice-cold lysis buffer [ $1 \mathrm{mM}$ dithiothreitol (DTT), $10 \mathrm{mM} \mathrm{NaCl}, 1.5 \mathrm{mM} \mathrm{MgCl}_{2}$, $20 \mathrm{mM}$ 4-(2-hydroxyethyl)-1-piperazineethanesulfonic acid (HEPES), 20\% glycerol, and $0.1 \%$ Triton X-100; pH 7.4] containing protease inhibitors. After a centrifugation at $12,000 \mathrm{~g}$ for $15 \mathrm{~min}$ at $4^{\circ} \mathrm{C}$, the supernatant was collected, and the protein concentration was measured using a bicinchoninic acid assay. Mitochondria were isolated according to the method as previously described (Trounce et al., 1996). Briefly, the supernatant was centrifuged at $8,000 \mathrm{~g}$ for $15 \mathrm{~min}$, and then was resuspended in isolation buffer $(210 \mathrm{mM}$ mannitol, $1 \mathrm{mM}$ EGTA, $70 \mathrm{mM}$ sucrose, and $5 \mathrm{mM}$ HEPES) and recentrifuged at $8,000 \mathrm{~g}$ for $15 \mathrm{~min}$. The resulting mitochondrial pellet was used for further analysis.

\section{Determination of the Oxidation Products}

Reactive oxygen species was determined as previously described (McMillan and Quadrilatero, 2011). Briefly, whole-muscle lysates or mitochondrial pellets were incubated with $5 \mu \mathrm{M}$ 2',7'-dichlorodihydrofluorescein diacetate (DCFH-DA; Invitrogen, Carlsbad, CA, United States) for $20 \mathrm{~min}$ at $37^{\circ} \mathrm{C}$ in the dark. The samples were analyzed using a microplate spectrofluorometer with an excitation and an emission wavelength of 490 and $535 \mathrm{~nm}$, respectively. Data were expressed as arbitrary units per milligram of protein. The superoxide radical contents were determined based on the methods as described (Azzi et al., 1975; Hong and Lee, 2009). Briefly, whole-muscle lysates were added to a reaction mixture containing $90 \mathrm{mM}$ succinate, $50 \mathrm{mM}$ phosphate buffer ( $\mathrm{pH} 7.5$ ), $30 \mathrm{nM} \mathrm{KCN}, 150 \mathrm{mM} \mathrm{KCl}$, and $0.3 \mathrm{mM}$ cytochrome $c$, and mixed in an oscillator for $5 \mathrm{~min}$. The absorbance was measured at a wavelength of $550 \mathrm{~nm}$. The hydrogen peroxide contents in the tissues were measured based on protocol as previously described (Jiang et al., 1992; Hong and Lee, 2009). Briefly, whole-muscle lysates were mixed with $100 \mathrm{mM}$ sorbitol, $100 \mu \mathrm{M}$ xylenol orange, $250 \mu \mathrm{M}$ ammonium 
ferrus sulfate, and $25 \mathrm{mM} \mathrm{H}_{2} \mathrm{SO}_{4}$. After incubation at $25^{\circ} \mathrm{C}$ for $30 \mathrm{~min}$, the absorbance at $560 \mathrm{~nm}$ was measured.

\section{Measurements of Oxidative Damage}

DNA was isolated from the skeletal muscles of the upper hindlimb using a DNA isolation kit obtained from Qiagen (Shanghai, China). The levels of 8-hydroxy-2'-deoxyguanosine (8-OHdG) were measured as previously described (Mecocci et al., 1999). The results were expressed as the ratio of nanomoles of 8-OHdG to nanomoles of 2 -deoxyguanosine $\times 10^{5}$. Whole-muscle lysates were used for the determination of malondialdehyde (MDA) and protein carbonyl by using specific enzyme-linked immunosorbent assay (ELISA) kits (NWK-MDA01 and NWK-PCK01, respectively; Northwest Life Science Specialties, Vancouver, WA, United States) according to the manufacturer's instructions. The MDA concentration of samples was determined by measuring the absorption at $532 \mathrm{~nm}$. The protein carbonyl was determined by measuring the absorbance at $450 \mathrm{~nm}$. Lipids were extracted from the skeletal muscles of the upper hindlimb using Folch solution, and the level of 8-isoprostane was determined using an ELISA kit (NWK-ISO01, Northwest Life Science Specialties) according to the manufacturer's instructions. The absorbance at $450 \mathrm{~nm}$ was measured after the addition of an acid stop solution which causing a color change to yellow.

\section{Measurement of Mitochondrial Oxidative Markers}

The activities of complex I (NADH ubiquinone reductase), complex II (succinate-coenzyme Q reductase), complex III (ubiquinol cytochrome c reductase), complex IV (cytochrome oxidase), and citrate synthase (CS) in the skeletal muscle were assessed spectrophotometrically using commercial Assay Kits (catalogue No BC0515, BC3230, BC3240, BC0945, and BC1064, respectively; Solarbio, Beijing, China) according to the manufacturer's instructions. The absorbance was measured at $340 \mathrm{~nm}$ for complex I, $605 \mathrm{~nm}$ for complex II, $550 \mathrm{~nm}$ for complex III, $550 \mathrm{~nm}$ for complex IV, and $412 \mathrm{~nm}$ for citrate synthase, respectively.

\section{Measurements of Antioxidant Enzyme}

The activities of catalase (CAT), manganese superoxide dismutase (MnSOD), and copper-zinc superoxide dismutase (CuZnSOD) in the whole-muscle lysates were measured using commercial ELISA kits (NWK-CAT01 and NWK-SOD02, respectively; Northwest Life Science Specialties) according to the manufacturer's instructions. CAT activity was measured by monitoring the consumption of the $\mathrm{H}_{2} \mathrm{O}_{2}$ substrate at $240 \mathrm{~nm}$. The activity measured using the original assay buffer reflects total superoxide dismutase (SOD) activity, whereas the activity measured in $\mathrm{KCN}$-containing assay buffer is only due to MnSOD activity. The absorbance was measured at $560 \mathrm{~nm}$.

\section{Determination of the Intracellular Glutathione Antioxidant System}

Reduced glutathione (GSH), oxidized glutathione (GSSG) content, and the activities of glutathione S-transferase (GST), glutathione reductase (GRD), and glutathione peroxidase (GPX) were determined using commercial ELISA kits (Item No 703002, 703302, 703202, and 703102, respectively; Cayman Chemical Company, Ann Arbor, Michigan, United States), according to the manufacturer's instructions. GSH content was calculated by measurement of the absorbance of 5-thio-2-nitrobenzoic acid at $405-414 \mathrm{~nm}$. Determination of GSSG was performed by prior GSH derivatization with 2-vinylpyridine using the same kit. Total GST activity was determined by measuring the absorbance at $340 \mathrm{~nm}$ after a conjugation of 1-chloro-2,4dinitrobenzene with reduced glutathione. GRD activity was determined by measuring the absorbance at $340 \mathrm{~nm}$ after oxidation of NADPH to $\mathrm{NADP}^{+}$. GPX activity was indirectly evaluated through a coupled reaction with GRD; the absorbance was measured at $340 \mathrm{~nm}$. Gcl activity was determined according to the method described in previous studies (White et al., 2003; Wu et al., 2009). Briefly, the skeletal muscles were homogenized in ice cold buffer $(0.25 \mathrm{M}$ sucrose, $20 \mathrm{mM}$ Tris, $20 \mathrm{mM}$ boric acid, $1 \mathrm{mM}$ EDTA, $1 \mathrm{mM} \mathrm{L}$-serine, and $\mathrm{pH} 7.4$ ), and a supernatant was obtained by centrifuging at $20,000 \mathrm{~g}$ for $30 \mathrm{~min}$ at $4^{\circ} \mathrm{C}$. The supernatant was mixed with $100 \mu \mathrm{l}$ cysteine and $100 \mu \mathrm{l}$ reaction cocktail $\left(40 \mathrm{mM} \mathrm{MgCl}_{2}, 2 \mathrm{mM}\right.$ EDTA, $20 \mathrm{mM}$ boric acid, $2 \mathrm{mM}$ L-serine, $400 \mathrm{mM}$ Tris, $40 \mathrm{mM}$ ATP, and $40 \mathrm{mM} \mathrm{L}$-glutamic acid). After incubation for $30 \mathrm{~min}$ at $37^{\circ} \mathrm{C}, 100 \mathrm{ml}$ of $200 \mathrm{mM} \mathrm{5}$-sulfosalicylic acid was added, and the supernatant was collected by a centrifugation at $2,000 \mathrm{~g}$ for $10 \mathrm{~min}$, which was used for the measurement of fluorescence intensities at an excitation wavelength of $485 \mathrm{~nm}$, and an emission wavelength of $538 \mathrm{~nm}$.

\section{qRT-PCR Analysis}

RNA was isolated from mixed upper hindlimb using TRIzoL reagent (Invitrogen Corporation, Carlsbad, CA, United States). One microgram of RNA from each sample was reversetranscribed to complementary DNA (cDNA) in 20- $\mu$ l reactions (Qiagen, Germantown, MD, United States). The resulting cDNA was used for $\mathrm{qPCR}$ using the primers shown in Supplementary Table 1 . Briefly, $1 \mu$ l of the cDNA template was added to a total volume of $10 \mu$ reaction solution containing $5 \mu \mathrm{l}$ SYBR Green mix (Qiagen), $3 \mu$ deionized $\mathrm{H}_{2} \mathrm{O}, 0.2 \mu \mathrm{l}$ Rox, and $0.4 \mu \mathrm{l}$ of the forward and reverse primers. Real-time PCR was performed using the ABI-Prism 7500 Sequence Detection System (Applied Biosystems) as instructed by the manufacturer. All the genes were normalized to the housekeeping gene, and the relative differences in gene expression among the groups were determined using the comparative $C_{\mathrm{t}}$ value method (Livak and Schmittgen, 2001).

\section{Protein Qualification by the Wes Simple Western System}

Protein expression extracted from samples of the skeletal muscles was qualified using the Wes Simple Western System (Proteinsimple, San Jose, CA, United States). Proteins were mixed with Proteinsimple mixed reagent and then loaded into Wes 25 -well plates. Primary antibodies $[\beta$-actin, lamin B, constitutive-androstane-receptor (CAR), nuclear-factor-erythroid2-related factor 2 (NRF2), and Keap1 (Kelch like ECH-associated 
protein 1), Abcam, Cambridge, MA, United States], secondary antibodies, and stacking gel and separation gel matrix were added according to the manufacturer's instructions. Results were collected using the Protein Simple software (Proteinsimple).

\section{Treadmill Testing}

Treadmill testing was performed as previously reported (Kadoguchi et al., 2015). In brief, after a 10-min adaption, the mouse was placed on the treadmill (Columbus Instruments, Columbus, $\mathrm{OH}$, United States) set at $6 \mathrm{~m} / \mathrm{min}$ and $0^{\circ}$ for $10 \mathrm{~min}$, after which, the inclination was set at $10^{\circ}$ and the speed was gradually increased by $2 \mathrm{~m} / \mathrm{min}$ every $2 \mathrm{~min}$ until the mouse reached exhaustion. Exhaustion was defined as spending at least $10 \mathrm{~s}$ on the shocker plate without trying to re-engage the treadmill. Work was defined as the product of the vertical running distance to exhaustion and body weight.

\section{Statistical Analysis}

Trial experiments or experiments done previously were used to determine sample size with adequate statistical power. Statistical analysis was performed by factorial ANOVA using a mixed procedure (PROCMIXED) with SAS software version 9.2 (SAS Institute Inc., Cary, NC, United States). All data were analyzed by a normality test, and variance was compared using a Bartlett's test. Differences between means were determined by using the Duncan multiple-comparison test. For 2-factor ANOVA, the PROC general linear model was used, with L-serine and doxorubicin as main factors. Data are presented as means \pm SEM. A value of $p<0.05$ was considered significant.

\section{RESULTS}

\section{Effects of Serine Administration on Oxidative Damage in the Skeletal Muscles of Mice}

As shown in Figure 1, doxorubicin exposure led to increased ROS contents in both the cellular and mitochondria, as compared with the controls (Figures 1A,D). This effect of doxorubicin was attenuated by serine pre-administration. Further study showed that doxorubicin treatment resulted in elevated levels of superoxide radical and hydrogen peroxide in skeletal muscles, which were markedly reduced by serine administration (Figures 1B,C). Consistently, doxorubicin treatment led to oxidative damage to DNA, protein, and lipid, as shown by increased contents of 8-OHdG, protein carbonyl, as well as MDA and 8-isoprostane, respectively (Figures $\mathbf{1 E}-\mathbf{H}$ ). These deleterious effects of doxorubicin were remarkably prevented by serine administration, indicating a protective effect of serine on oxidative damage in skeletal muscles of doxorubicin-challenged mice.

\section{Effects of Serine Administration on Mitochondrial Respiratory Complex in the Skeletal Muscles of Mice}

As shown, doxorubicin treatment led to enhanced activity of complex I (Figure 2A), without affecting the activities of complex II, complex III, or complex IV in skeletal muscles (Figures 2B-D). The effect of doxorubicin on activity of complex I was prevented by serine administration. Also, we found that doxorubicin treatment led to decreased activity of citrate synthase (Figure 2E), while increasing mRNA level of Hspd1 (Figure 2F), as compared with

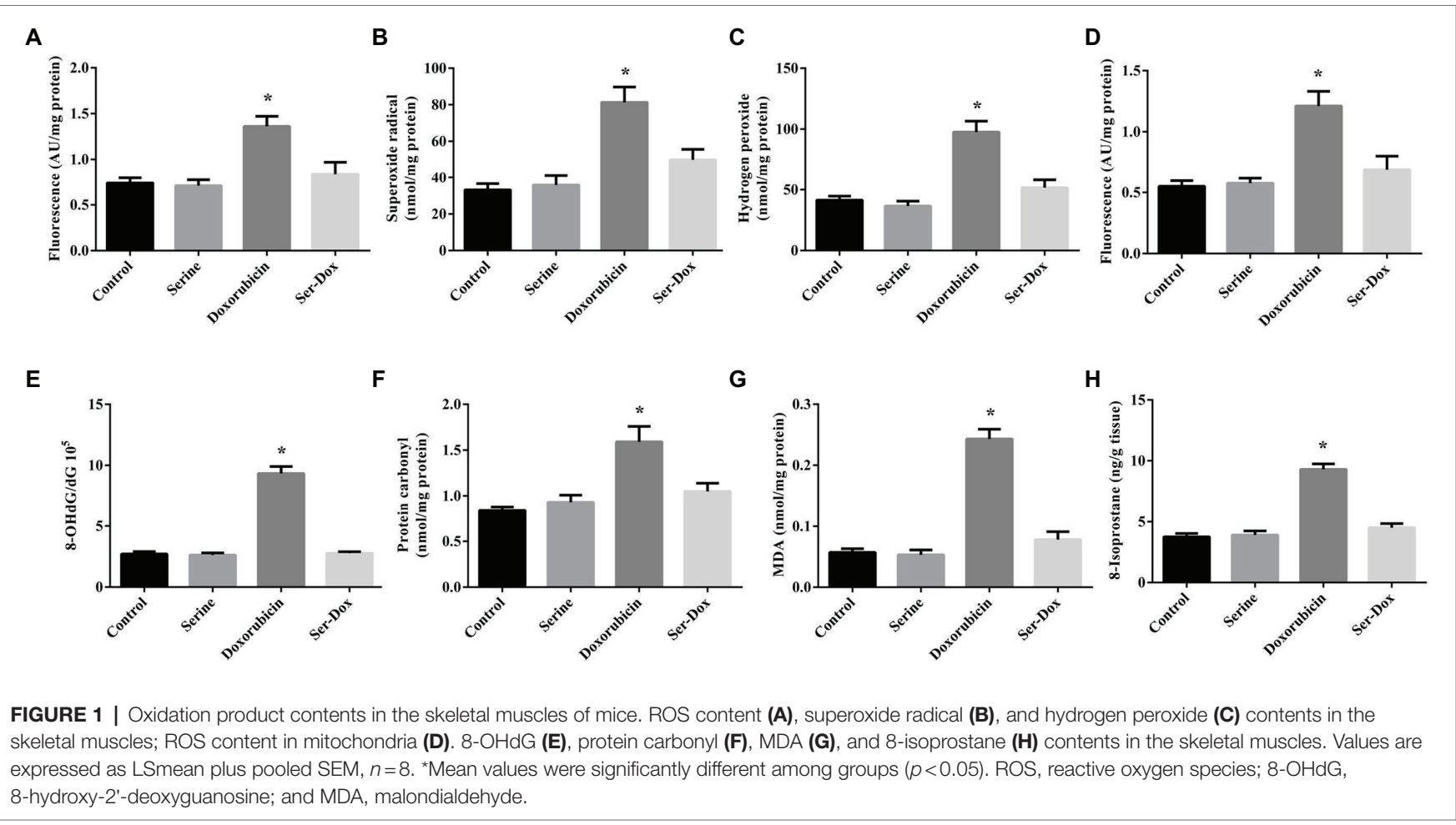


the controls. These results indicated that serine mainly regulated activity of respiratory complex I in doxorubicin-challenged mice.

\section{Effects of Serine Administration on the Activities of Antioxidant Enzymes and Glutathione Content in the Skeletal Muscles of Mice}

Compared with the control, the activities of CAT, CuZnSOD, and MnSOD were significantly reduced following doxorubicin treatment, which were abrogated by serine pre-administration (Figures 3A-C). To explore an implication of non-enzymatic antioxidant system in our animal model, the activity of glutathione was measured. As indicated, GSH content, the ratio of GSH to GSSG, the activities of GPX, GRD, and GST were significantly reduced by doxorubicin treatment (Figures 3D-H). Interestingly, these alterations triggered by doxorubicin were abolished by serine pre-administration. In contrast, the mRNA levels for genes involved in the thioredoxin and peroxiredoxin system, including TRX, TRR, PRX1, and PRX3, were not affected by doxorubicin, serine, or doxorubicin plus serine treatment (Supplemental Figure 1), thus excluding an involvement of these genes in our animal model.

\section{Effects of Serine Administration on the Amino Acid Contents and GSH Synthesis in the Skeletal Muscles and Livers of Mice}

Serine, as well as glycine which could be used for endogenous synthesis of serine, were measured in the skeletal muscle of mice. The contents of serine and glycine (Supplementary Figures 2A,B) increased significantly following doxorubicin treatment. However, orally serine administration had no effect on the amino acids changes in the skeletal muscles of the doxorubicin-treated mice. In addition, we determined serine and glycine in the liver and the contents were decreased significantly in the liver, which were significantly attenuated by the presence of serine (Supplementary Figures 2D,E). To explore whether the beneficial effect of serine supplementation was mediated by GSH synthesis in the muscle, we determined the content of cysteine (a key substrate for GSH synthesis) and activity of Gcl (an enzyme for GSH synthesis), and found that both of them were not affected by serine supplementation in doxorubicinchallenged mice (Supplementary Figures 2C, 3A). Considering that the liver is the largest organ and main source of GSH synthesis, we speculated that the increase of GSH content in the skeletal muscle might due to an elevated production of GSH in the liver, which was confirmed by both the content of substrates (glycine and cysteine), Gcl activity, and production of GSH in the liver tissues of doxorubicin-challenged mice (Supplementary Figures 2D-F, 3C,D).

\section{Effects of Serine Administration on NRF2/CAR Signaling in the Skeletal Muscles of Mice}

Compared with control, doxorubicin treatment led to decreased abundance of nuclear NRF2, as well as its downstream target CAR. This effect of doxorubicin was prevented by serine

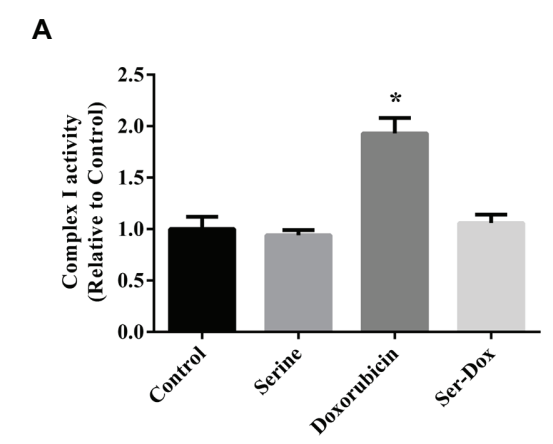

D

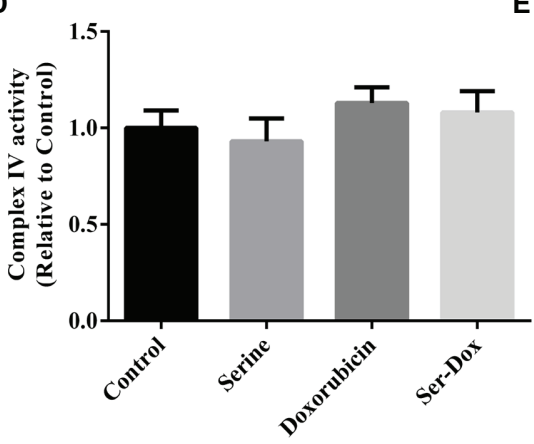

B
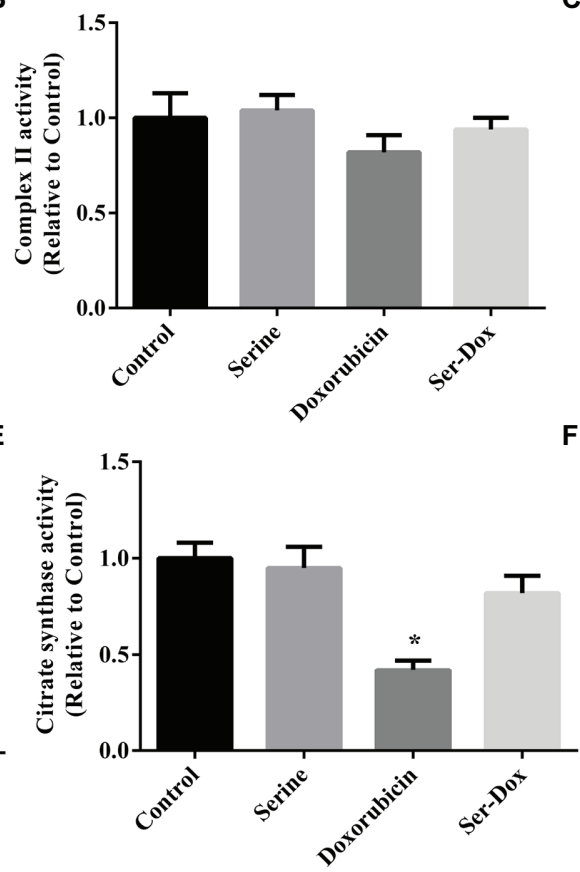

C
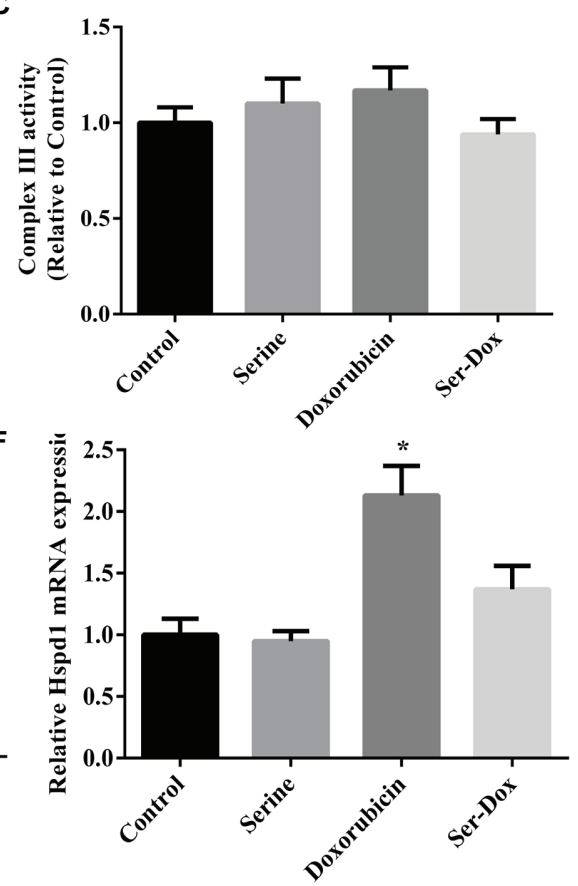

FIGURE 2 | Mitochondrial respiratory complex in the skeletal muscles of mice. Activity of complex I (A), complex I| (B), complex III (C), complex IV (D), citrate synthase (E), and mRNA expression of Hspd1 (F) in the skeletal muscles. Values are expressed as LSmean plus pooled SEM, $n=8$. ${ }^{\star}$ Mean values were significantly different among groups $(p<0.05)$. Hspd1, heat shock protein 1. 


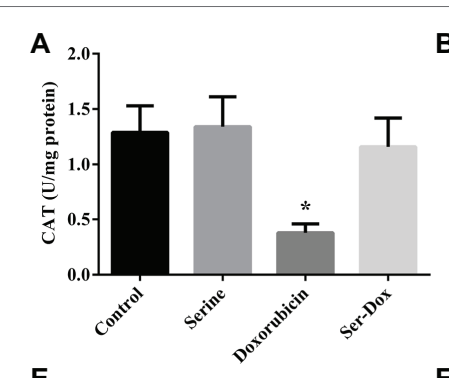

E

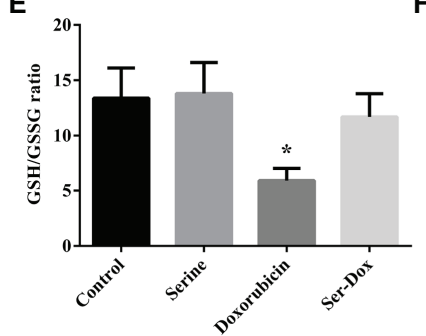

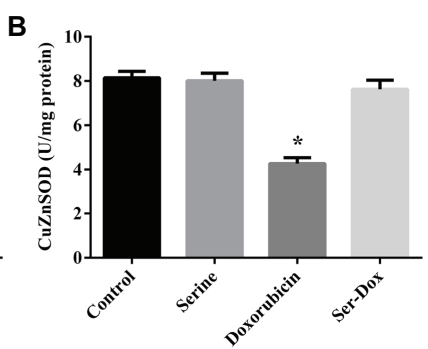

F

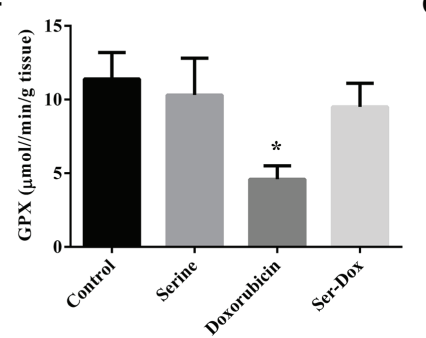

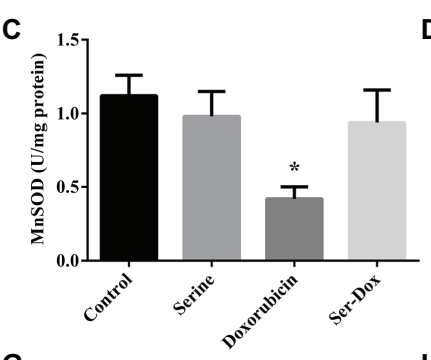

G

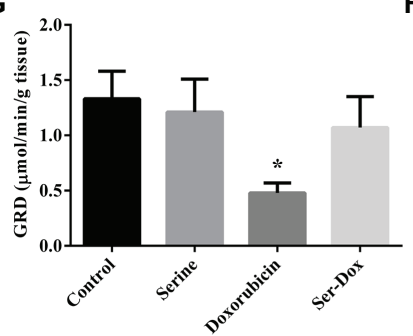

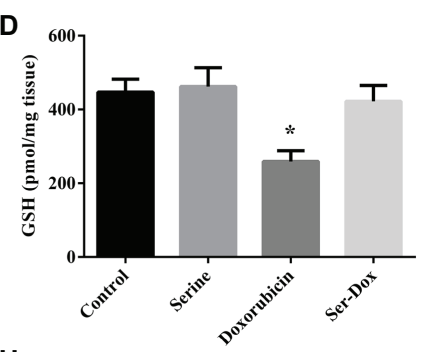

H

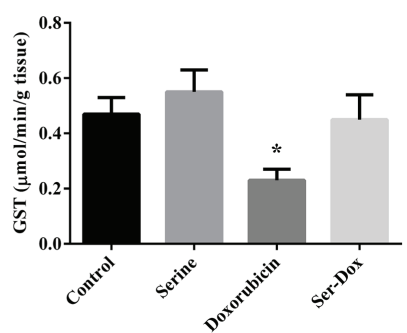

FIGURE 3 | The activities of antioxidant enzymes and glutathione content in the skeletal muscles of mice. CAT (A), CuZnSOD (B), and MnSOD (C) activities in the skeletal muscles; GSH content (D), GSH/GSSG ratio (E), GPX (F), GRD (G), and GST (H) activities in the skeletal muscles. Values are expressed as LSmean plus pooled SEM, $n=8$. *Mean values were significantly different among groups $(p<0.05)$. CAT, catalase; MnSOD, manganese superoxide dismutase; CuZnSOD, copper-zinc superoxide dismutase; GSH, reduced glutathione; GSSG, oxidized glutathione; GST, glutathione S-transferase; GRD, glutathione reductase; and GPX, glutathione peroxidase.

administration (Figures $\mathbf{4 A}-\mathbf{C}$ ). Doxorubicin-induced downregulation of Keap1, a regulator of NRF2 signaling, which was abolished by serine administration (Figure 4D). Protein abundance of total NRF2 and total CAR was not affected by doxorubicin, serine administration, or doxorubicin plus serine co-treatment (Figures 4E,F).

\section{Effects of Serine Administration on the Exercise Capacity of Mice}

To evaluate a functional role of serine administration on doxorubicin-induced skeletal muscle dysfunction, exercise capacity was examined by using the treadmill assay. As shown in Figure 5, mice exposed to doxorubicin had reduced exercise capacity as shown by shorter running time, shorter running distance, as well as a lower excise performance (Figures $\mathbf{5 A}-\mathbf{C}$ ), indicating a deleterious effect of doxorubicin on muscle function. Intriguingly, serine administration abolished the effect of doxorubicin on skeletal muscle, supporting a beneficial effect in muscle tissue of mice. Serine pre-administration had no effect on exercise capacity in mice.

\section{DISCUSSION}

In the present study, we found that serine administration reduced doxorubicin-induced oxidative damage, as evidenced by reduced protein, DNA, and lipid oxidation in skeletal muscle, as well as increased exercise capacity. This beneficial effect of serine was associated with reduced ROS accumulation, increased activity of the antioxidant enzymes, such as CAT, MnSOD, CuZnSOD, and increased intracellular GSH level, and upregulation of NRF2 signaling.
Doxorubicin is a chemotherapeutic drug for various malignances, including solid tumor and hemorrhage tumors. However, clinical application of doxorubicin leads to unexpected side effects on the muscle, therefore reducing quality of life and physical activity of patients. Oxidative damage is one of the major mediators that implicated in and contribute to muscle dysfunction in response to doxorubicin treatment. Nutritional strategy to reduce oxidative damage and restore muscle function is of great significance and attracts more and more attention. Our recent studies have shown that serine supplementation abrogates lipopolysaccharide-induced damage in animals and intestinal epithelial cells (Zhou et al., 2017b, 2018a). It remains unknown whether serine can protect mice against doxorubicin-induced skeleton muscle weakness.

In the present study, mice fed an AIN-93 diet supplemented with or without $1.5 \mathrm{~g} / \mathrm{kgBW}^{-1}$ day $^{-1}$ serine were subjected to doxorubicin to induce oxidative damage in the skeleton muscle. Serine administration had no effect on feed intake and body weight gain during the whole experimental period (data not shown). In agreement with previous studies (Smuder et al., 2011; Min et al., 2015), doxorubicin injection resulted in enhanced oxidative damage of lipid, DNA, and proteins in skeleton muscle and reduced exercise capacity. Intriguingly, these alterations were not observed in serine-pretreated mice, indicating a preventive effect on oxidative damage in skeleton muscle.

To maintain intracellular homeostasis, the cells have evolved both enzymatic anti-oxidative systems, such as MnSOD, CuZnSOD, and CAT, as well as non-enzymatic anti-oxidative systems, including GSH and TRXN. In the present study, we found that doxorubicin exposure led to decreased activity of MnSOD, CuZnSOD, and CAT, indicating an impairment of enzymatic anti-oxidative capacity in response to doxorubicin. Interestingly, these effects of doxorubicin were abolished by serine pretreatment, indicating a regulatory 

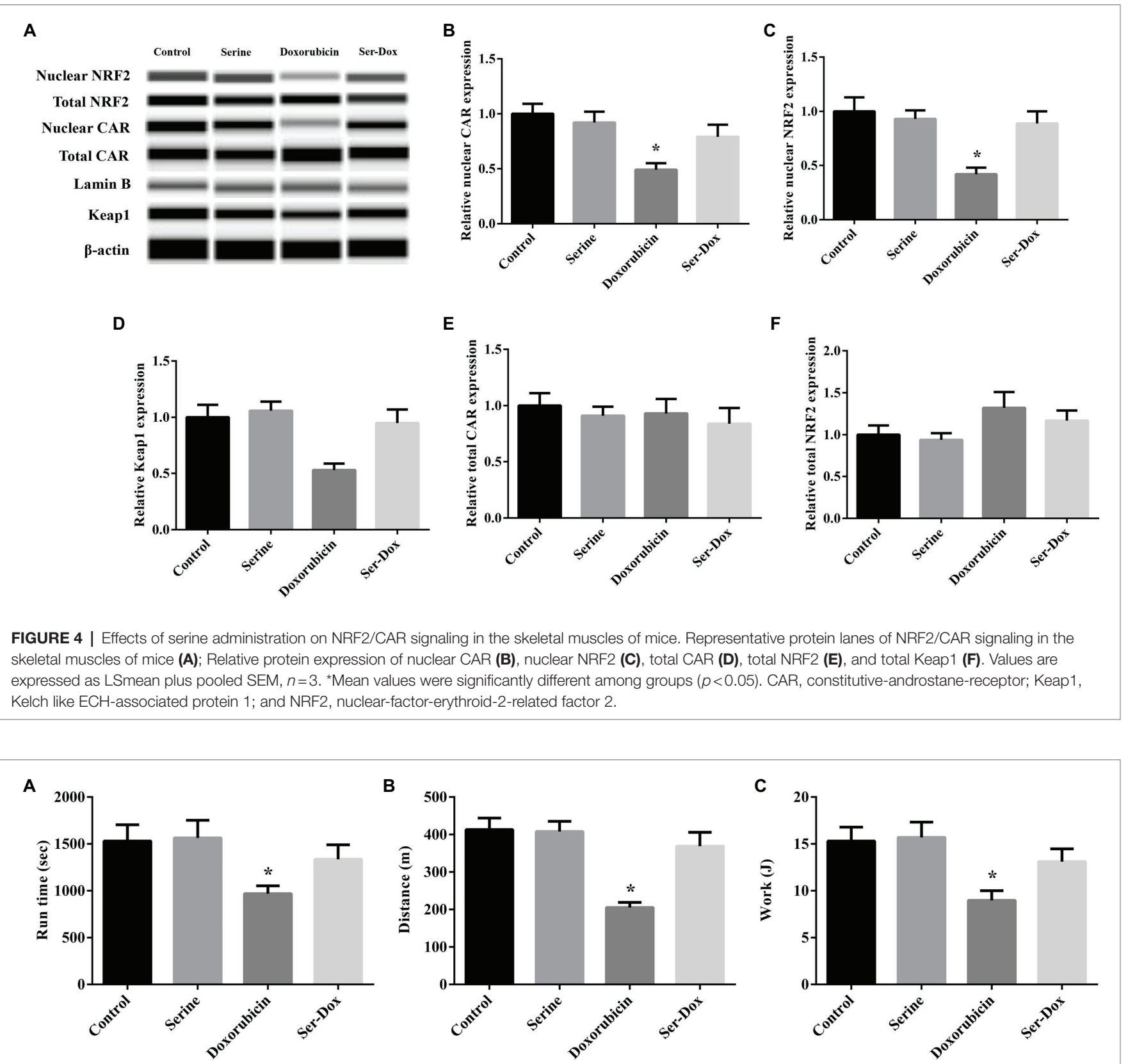

FIGURE 5 | Effects of serine administration on the exercise capacity of mice. (A) Run time, (B) distance, and (C) excise performance were evaluated. Values are expressed as LSmean plus pooled SEM, $n=8$. *Mean values were significantly different among groups $(p<0.05)$.

effect of serine on anti-oxidative signaling. For the non-enzymatic antioxidant system, we found that GSH, but no TRXN level, were decreased by doxorubicin, thus implicating in oxidative damage in muscle tissues. GSH is one of the most abundant anti-oxidative tripeptides in the body (Ji et al., 1992). Our previous study has shown that glycine supplementation leads to increased GSH level in small intestine and serum, thus enhancing antioxidative capacity in piglet (Wang et al., 2014). Similar result was observed in skeletal muscles of doxorubicin-challenged mice. However, neither the content of cysteine (a key substrate for GSH synthesis) nor Gcl activity was affected by serine in the skeletal muscles of mice. Of note, we found that serine administration resulted in increased levels of serine, glycine, cysteine, and GSH, as well as an enhanced Gcl activity in the liver of doxorubicin-challenged mice. The liver is the body's largest organ and the main source of GSH (DeLeve and Kaplowitz, 1991). The elevated production of GSH in the liver might contribute to an increased GSH content in the skeletal muscle, thus exerting a beneficial effect on oxidative damage. Availability of substrates and activity of enzymes implicated in GSH synthesis have been reported as critical factors for production of GSH in various conditions (Zhou et al., 2017a, 2018b), which might be one of 
the reasons for the elevated GSH in the skeletal muscle of mice (DeLeve and Kaplowitz, 1991). In addition to de novo synthesis of GSH, GRD can catalyze the GSSG to its reduced form and contribute to increased GSH level. Serine administration abrogated doxorubicin-induced downregulation of GRD, and led to increased GSH as observed in our study. These results indicated a regulatory effect of serine on intracellular redox homeostasis by enhancing GSH production (Meister and Anderson, 1983).

Mitochondria are the primary source for ROS production in skeletal muscle (Brand, 2010). Increased activities of electrontransport-complex of complex I, complex II-III, and complex $\mathrm{IV}$, as well as decreased citrate synthase activity have been associated with elevated ROS level and oxidative damage in cells (Tarry-Adkins et al., 2016). In our study, we found that activity of complex I, instead of complexes II, III, and IV, was increased in response to doxorubicin administration, indicating that complex I was more sensitive to doxorubicin than other complexes. This result is agreement with previous study (Smuder et al., 2011). Importantly, the increased activity of complex I in the skeletal muscle and decreased activity of citrate synthase were significantly attenuated by serine administration. Additionally, doxorubicin-induced gene expression of Hspd1, a gene involved in stress response of the mitochondria, was partially prevented by serine. This is the first study showing that serine administration prevented the effect of doxorubicin on oxidative damage in muscle of mice.

The NRF2 is a master transcriptional factor that regulates the antioxidant defense systems. Under normal condition, the activity of NRF2 is restricted due to formation of the NRF2Keap1 complex in cytosol (Chan et al., 2001). In response to oxidative stress, NRF2 is released and translocated to the nucleus, where it can trans-activate androstane receptor (CAR) signaling, which in turn, activates downstream anti-oxidative targets implicated in glutathione antioxidant system (Chan et al., 2001; He et al., 2017; Liu et al., 2018). To explore an involvement of the NRF2/CAR signaling pathway in doxorubicininduced oxidative damage in skeletal muscle of mice, Western blot analysis was conducted to determine the protein abundance of Keap1, NRF2, and CAR. As expected, we found that doxorubicin exposure led to suppressed NRF2/CAR signaling, which was abrogated by serine administration, indicating a regulatory effect of serine and contribution to the beneficial effect.

\section{CONCLUSION}

In conclusion, using doxorubicin-treated mice as a model of skeletal muscle weakness, we showed, for the first time, that

\section{REFERENCES}

Azzi, A., Montecucco, C., and Richter, C. (1975). The use of acetylated ferricytochrome $\mathrm{c}$ for the detection of superoxide radicals produced in biological membranes. Biochem. Biophys. Res. Commun. 65, 597-603. doi: 10.1016/S0006-291X(75)80188-4

Brand, M. D. (2010). The sites and topology of mitochondrial superoxide production. Exp. Gerontol. 45, 466-472. doi: 10.1016/j.exger.2010.01.003 serine administration prevented doxorubicin-induced oxidative damage by reducing ROS in the skeletal muscle of mice. This beneficial effect was associated with enhanced anti-oxidative capacity include both enzymatic and non-enzymatic systems in which NRF2-CAR signaling is implicated. Our study provides new insights on the functional role of serine in regulating intracellular redox homeostasis. Supplementation of serine might be a nutritional strategy to ameliorate skeletal muscle atrophy, a side effect observed in patients receiving doxorubicin treatment.

\section{DATA AVAILABILITY STATEMENT}

The raw data supporting the conclusions of this article will be made available by the authors, without undue reservation.

\section{ETHICS STATEMENT}

The animal study was reviewed and approved by Committee of the Institute of Subtropical Agriculture, the Chinese Academy of Science.

\section{AUTHOR CONTRIBUTIONS}

JC and XZ performed the experiment, analyzed the data, and wrote the manuscript. HJ assisted the research and data analysis. $\mathrm{XZ}$ and ZW designed the study. ZW reviewed and edited the manuscript and had primary responsibility for final content. All authors contributed to the article and approved the submitted version.

\section{FUNDING}

This study was supported by the National Natural Science Foundation of China (Nos. 31625025, 31572410, and 31272451), the "111" Project (B16044), National Key R\&D Program of China (2018YFD0500405 and 2018YFD0501003), Natural Science Foundation of Hunan Province (2017JJ3373), and the earmarked fund for China Agriculture Research System (CARS-35).

\section{SUPPLEMENTARY MATERIAL}

The Supplementary Material for this article can be found online at: https://www.frontiersin.org/articles/10.3389/fphys.2021.727093/ full\#supplementary-material

Cao, G. T., Tao, F., Xin, L., Li, Z. M., and Zhou, X. H. (2018). Effects of maternal serine supplementation on high-fat diet-induced oxidative stress and epigenetic changes in promoters of glutathione synthesis-related genes in offspring. J. Funct. Foods 47, 316-324. doi: 10.1016/j.jff.2018. 05.067

Chan, K., Han, X. D., and Kan, Y. W. (2001). An important function of Nrf2 in combating oxidative stress: detoxification of acetaminophen. Proc. Natl. Acad. Sci. U. S. A. 98, 4611-4616. doi: 10.1073/pnas.081082098 
DeLeve, L. D., and Kaplowitz, N. (1991). Glutathione metabolism and its role in hepatotoxicity. Pharmacol. Ther. 52, 287-305. doi: 10.1016/0163-7258(91)90029-L

Fabris, S., and MacLean, D. A. (2018). Doxorubicin chemotherapy affects the intracellular and interstitial free amino acid pools in skeletal muscle. PLoS One 13:e0195330. doi: 10.1371/journal.pone.0195330

Gaucher, C., Boudier, A., Bonetti, J., Clarot, I., Leroy, P., and Parent, M. (2018). Glutathione: antioxidant properties dedicated to nanotechnologies. Antioxidants 7:62. doi: $10.3390 /$ antiox7050062

Gilliam, L. A. A., Fisher-Wellman, K. H., Lin, C. T., Maples, J. M., Cathey, B. L., and Neufer, P. D. (2013). The anticancer agent doxorubicin disrupts mitochondrial energy metabolism and redox balance in skeletal muscle. Free Radic. Biol. Med. 65, 988-996. doi: 10.1016/j.freeradbiomed.2013.08.191

He, L. Q., Zhang, H. W., and Zhou, X. H. (2018). Weanling offspring of dams maintained on serine deficient diet are vulnerable to oxidative stress. Oxidative Med. Cell. Longev. 2018:8026496. doi: 10.1155/2018/8026496

He, L., Zhou, X., Huang, N., Li, H., Li, T., Yao, K., et al. (2017). Functions of pregnane X receptor in self-detoxification. Amino Acids 49, 1999-2007. doi: $10.1007 /$ s00726-017-2435-0

Hong, J. H., and Lee, I. S. (2009). Effects of Artemisia capillaris ethyl acetate fraction on oxidative stress and antioxidant enzyme in high-fat diet induced obese mice. Chem. Biol. Interact. 179, 88-93. doi: 10.1016/j.cbi.2008.12.002

Ji, L. L., Fu, R., and Mitchell, E. W. (1992). Glutathione and antioxidant enzymes in skeletal muscle: effects of fiber type and exercise intensity. J. Appl. Physiol. 73, 1854-1859. doi: 10.1152/jappl.1992.73.5.1854

Jiang, Z. Y., Hunt, J. V., and Wolff, S. P. (1992). Ferrous ion oxidation in the presence of xylenol orange for detection of lipid hydroperoxide in low density lipoprotein. Anal. Biochem. 202, 384-389. doi: 10.1016/0003-2697(92)90122-N

Kadoguchi, T., Kinugawa, S., Takada, S., Fukushima, A., Furihata, T., Homma, T., et al. (2015). Angiotensin II can directly induce mitochondrial dysfunction, decrease oxidative fibre number and induce atrophy in mouse hindlimb skeletal muscle. Exp. Physiol. 100, 312-322. doi: 10.1113/expphysiol.2014.084095

Kalhan, S. C., and Hanson, R. W. (2012). Resurgence of serine: an often neglected but indispensable amino acid. J. Biol. Chem. 287, 19786-19791. doi: $10.1074 /$ jbc.R112.357194

Kavazis, A. N., Smuder, A. J., and Powers, S. K. (2014). Effects of short-term endurance exercise training on acute doxorubicin-induced FoxO transcription in cardiac and skeletal muscle. J. Appl. Physiol. 117, 223-230. doi: 10.1152/ japplphysiol.00210.2014

Liu, N., Ma, X., Luo, X., Zhang, Y., He, Y., Dai, Z., et al. (2018). L-glutamine attenuates apoptosis in porcine enterocytes by regulating glutathione-related redox homeostasis. J. Nutr. 148, 526-534. doi: 10.1093/jn/nxx062

Livak, K. J., and Schmittgen, T. D. (2001). Analysis of relative gene expression data using real-time quantitative PCR and the 2(-Delta Delta C(T)) method. Methods 25, 402-408. doi: 10.1006/meth.2001.1262

McMillan, E. M., and Quadrilatero, J. (2011). Differential apoptosis-related protein expression, mitochondrial properties, proteolytic enzyme activity, and DNA fragmentation between skeletal muscles. Am. J. Physiol. Regul. Integr. Comp. Physiol. 300, R531-R543. doi: 10.1152/ajpregu.00488.2010

Mecocci, P., Fano, G., Fulle, S., MacGarvey, U., Shinobu, L., Polidori, M. C., et al. (1999). Age-dependent increases in oxidative damage to DNA, lipids, and proteins in human skeletal muscle. Free Radic. Biol. Med. 26, 303-308. doi: $10.1016 /$ S0891-5849(98)00208-1

Meister, A., and Anderson, M. E. (1983). Glutathione. Annu. Rev. Biochem. 52, 711-760. doi: 10.1146/annurev.bi.52.070183.003431

Metcalf, J. S., Dunlop, R. A., Powell, J. T., Banack, S. A., and Cox, P. A. (2018). L-serine: a naturally-occurring amino acid with therapeutic potential. Neurotox. Res. 33, 213-221. doi: 10.1007/s12640-017-9814-x

Min, K., Kwon, O. S., Smuder, A. J., Wiggs, M. P., Sollanek, K. J., Christou, D. D., et al. (2015). Increased mitochondrial emission of reactive oxygen species and calpain activation are required for doxorubicin-induced cardiac and skeletal muscle myopathy. J. Physiol. 593, 2017-2036. doi: 10.1113/jphysiol.2014.286518

Reeves, P. G., Nielsen, F. H., and Fahey, G. C. Jr. (1993). AIN-93 purified diets for laboratory rodents: final report of the American Institute of Nutrition ad hoc writing committee on the reformulation of the AIN-76A rodent diet. J. Nutr. 123, 1939-1951. doi: 10.1093/jn/123.11.1939

Schwartz, A. L. (2000). Daily fatigue patterns and effect of exercise in women with breast cancer. Cancer Pract. 8, 16-24. doi: 10.1046/j.1523-5394.2000.81003.x

Sim, W. C., Yin, H. Q., Choi, H. S., Choi, Y. J., Kwak, H. C., Kim, S. K., et al. (2015). L-serine supplementation attenuates alcoholic fatty liver by enhancing homocysteine metabolism in mice and rats. J. Nutr. 145, 260-267. doi: $10.3945 /$ jn.114.199711

Smuder, A. J., Kavazis, A. N., Min, K., and Powers, S. K. (2011). Exercise protects against doxorubicin-induced oxidative stress and proteolysis in skeletal muscle. J. Appl. Physiol. 110, 935-942. doi: 10.1152/japplphysiol.00677.2010

Songbo, M., Lang, H., Xinyong, C., Bin, X., Ping, Z., and Liang, S. (2019). Oxidative stress injury in doxorubicin-induced cardiotoxicity. Toxicol. Lett. 307, 41-48. doi: 10.1016/j.toxlet.2019.02.013

Tarry-Adkins, J. L., Fernandez-Twinn, D. S., Chen, J. H., Hargreaves, I. P., Neergheen, V., Aiken, C. E., et al. (2016). Poor maternal nutrition and accelerated postnatal growth induces an accelerated aging phenotype and oxidative stress in skeletal muscle of male rats. Dis. Model. Mech. 9, 1221-1229. doi: $10.1242 / \mathrm{dmm} .026591$

Trounce, I. A., Kim, Y. L., Jun, A. S., and Wallace, D. C. (1996). Assessment of mitochondrial oxidative phosphorylation in patient muscle biopsies, lymphoblasts, and transmitochondrial cell lines. Methods Enzymol. 264, 484-509.

van Norren, K., van Helvoort, A., Argiles, J. M., van Tuijl, S., Arts, K., Gorselink, M., et al. (2009). Direct effects of doxorubicin on skeletal muscle contribute to fatigue. Br. J. Cancer 100, 311-314. doi: 10.1038/sj.bjc.6604858

Wang, W., Wu, Z., Lin, G., Hu, S., Wang, B., Dai, Z., et al. (2014). Glycine stimulates protein synthesis and inhibits oxidative stress in pig small intestinal epithelial cells. J. Nutr. 144, 1540-1548. doi: 10.3945/jn.114.194001

White, C. C., Viernes, H., Krejsa, C. M., Botta, D., and Kavanagh, T. J. (2003). Fluorescence-based microtiter plate assay for glutamate-cysteine ligase activity. Anal. Biochem. 318, 175-180. doi: 10.1016/S0003-2697(03)00143-X

Wu, H., White, C. C., Isanhart, J. P., McBride, T. J., Kavanagh, T. J., and Hooper, M. J. (2009). Optimization and application of glutamate cysteine ligase measurement in wildlife species. Ecotoxicol. Environ. Saf. 72, 572-578. doi: 10.1016/j.ecoenv.2008.03.001

Yamamoto, Y., Hoshino, Y., Ito, T., Nariai, T., Mohri, T., Obana, M., et al. (2008). Atrogin-1 ubiquitin ligase is upregulated by doxorubicin via p38-MAP kinase in cardiac myocytes. Cardiovasc. Res. 79, 89-96. doi: 10.1093/cvr/cvn076

Zhou, X., He, L., Wu, C., Zhang, Y., Wu, X., and Yin, Y. (2017a). Serine alleviates oxidative stress via supporting glutathione synthesis and methionine cycle in mice. Mol. Nutr. Food Res. 61. doi: 10.1002/mnfr.201700262

Zhou, X. H., He, L. Q., Zuo, S. N., Zhang, Y. M., Wan, D., Long, C. M., et al. (2018b). Serine prevented high-fat diet-induced oxidative stress by activating AMPK and epigenetically modulating the expression of glutathione synthesis-related genes. Biochim. Biophys. Acta Mol. Basis Dis. 1864, 488-498. doi: 10.1016/j.bbadis.2017.11.009

Zhou, X. H., Zhang, Y. M., He, L. Q., Wan, D., Liu, G., Wu, X., et al. (2017b). Serine prevents LPS-induced intestinal inflammation and barrier damage via p53-dependent glutathione synthesis and AMPK activation. J. Funct. Foods 39, 225-232. doi: 10.1016/j.jff.2017.10.026

Zhou, X. H., Zhang, H. W., He, L. Q., Wu, X., and Yin, Y. L. (2018c). Longterm L-serine administration reduces food intake and improves oxidative stress and Sirt1/NF kappa B signaling in the hypothalamus of aging mice. Front. Endocrinol. 9:476. doi: 10.3389/fendo.2018.00476

Zhou, X., Zhang, Y., Wu, X., Wan, D., and Yin, Y. (2018a). Effects of dietary serine supplementation on intestinal integrity, inflammation and oxidative status in early-weaned piglets. Cell. Physiol. Biochem. 48, 993-1002. doi: $10.1159 / 000491967$

Conflict of Interest: The authors declare that the research was conducted in the absence of any commercial or financial relationships that could be construed as a potential conflict of interest.

Publisher's Note: All claims expressed in this article are solely those of the authors and do not necessarily represent those of their affiliated organizations, or those of the publisher, the editors and the reviewers. Any product that may be evaluated in this article, or claim that may be made by its manufacturer, is not guaranteed or endorsed by the publisher.

Copyright $(2021$ Chen, Zhou, Jia and Wu. This is an open-access article distributed under the terms of the Creative Commons Attribution License (CC BY). The use, distribution or reproduction in other forums is permitted, provided the original author(s) and the copyright owner(s) are credited and that the original publication in this journal is cited, in accordance with accepted academic practice. No use, distribution or reproduction is permitted which does not comply with these terms. 